\title{
DADDY'S CAR: LA INTELIGENCIA ARTIFICIAL COMO HERRAMIENTA FACILITADORA DE DERECHOS DE AUTOR
}

\author{
JAIME ALBERTO DÍAZ-LIMÓN*
}

\section{INTRODUCCIÓN}

En el año de 1982 el séptimo arte engalanó sus pantallas con el estreno de Blade Runner-de Ridley Scott-, obra de ciencia ficción que no solo marcó la industria cinematográfica sino que ha inspirado otras obras audiovisuales, así como diversas obras literarias relacionadas con el mundo de la inteligencia artificial. La película está parcialmente basada en la novela del estadounidense Philip Kindred Dick ¿Sueñan los androides con ovejas eléctricas? (1968). Las casi dos horas de la cinta describen un universo en que la ingeniería genética ha facilitado las labores complejas y peligrosas de los humanos, para dejar todo en manos de la inteligencia artificial incorporada en seres denominados replicantes. Estos fueron creados con la intención de "replicar" diversas características humanas, sin embargo, su función principal era mejorar lo que cualquier humano podría realizar, aun en su máximo potencial. El climax de la cinta reside en el soberbio parlamento pronunciado por el actor neerlandés Rutger Oelsen Hauer en su papel del replicante Roy Batty; justo después de salvar la vida de su persecutor:

He visto cosas que ustedes nunca hubieran podido imaginar, naves de combate en llamas más allá de Orión. He visto relámpagos resplandeciendo en la oscuridad cerca

\footnotetext{
* Catedrático y conferencista en materia de Propiedad Intelectual, Derecho Informático y Juicios Contencioso Administrativos en Línea. Profesor titular de Derecho Informático y Propiedad Intelectual en la Facultad de Derecho de la Barra Nacional de Abogaados de México y titular de diversos talleres, seminarios y diplomados en la Universidad Autónoma Metropolitana Azcapotzalco. Licenciado en Derecho por la Universidad Autónoma Metropolitana y magíster en Derecho Administrativo y Fiscal por la Facultad de Derecho de la Barra Nacional de Abogados de México. Contacto: [legal_limon@outlook.com]. Fecha de recepción: 10 de septiembre de 2016. Fecha de aceptación: 30 de noviembre de 2016. Para citar el artículo: Díaz-Limón, J. A. Daddy's Car: la inteligencia artificial como herramienta facilitadora de derechos de autor. Revista La Propiedad Inmaterial n. ${ }^{\circ}$ 22, Universidad Externado de Colombia, julio-diciembre 2016, pp. 83-100. Dor: http:// dx.doi.org/10.18601/16571959.n22.06.
} 
de la entrada de Tannhäuser. Todos estos momentos se perderán... en el tiempo... igual que lágrimas... en la lluvia. Llegó la hora de morir.

Para algunos expertos en física e inteligencia artificial (como Sidney Perkiwitz y Michio Kaku), el reputado parlamento demuestra la quintaesencia de la evolución humana, sus logros, su grandeza y su decadencia ${ }^{1}$, pues no sólo permite entender que las creaciones del hombre, como las máquinas, podrían alcanzar un nivel igual o superior de conciencia ${ }^{2}$, sino que además plantea dudas fundamentales relativas a la capacidad humana y a los límites que una inteligencia computarizada podría fácilmente superar: ¿las máquinas pueden hacer las cosas mejor que el hombre?, ¿la inteligencia artificial podría adquirir un nivel propio de conciencia universal?, ¿es acaso pertinente u ortodoxo cuestionar si la inteligencia artificial podría considerarse autónoma y "humana"?

El objeto del presente trabajo de investigación no consiste en la construcción metalingüística de un concepto jurídico de inteligencia artificial, ni pretende aportar argumentos a favor de las teorías de la conspiración o tecnoapocalípticas que han inundado las novelas de ciencia ficción y obras cinematográficas de los últimos lustros. Nuestro hilo negro de Ariadna es la publicación realizada el pasado 19 de septiembre de 2016 por el laboratorio de búsqueda sONY CSL y la Universidad Pierre and Marie Curie de París, a través de la plataforma Flow Machines, cuyo eslogan es "Artificial Intelligence for the future of music" (Inteligencia Artificial para el futuro de la música) ${ }^{3}$, por medio de la cual afirmaron haber creado y compuesto con inteligencia artificial (en adelante "IA") dos canciones enteras de pop. Lo anterior fue posible gracias a que el software de referencia aprendió (léase, para fines pragmáticos, almacenó) al menos trece mil distintas formas de escritura y lectura de partituras (melodía, armonía), de estilos y composiciones (principalmente jazz y рор); almacenando toda esa información en una base de datos (Big Data $)$ denominada Lead Sheet Data Base (LSDB). Bajo tales términos es posible "explotar

1 Kaku, Michio. La física del futuro. Cómo la ciencia determina el destino de la humanidad y nuestra vida cotidiana en el siglo XXI, disponible en: https://issuu.com/mazzymazzy/ docs/la_f__sica_del_futuro._michio_kaku. En particular, este gran trabajo de investigación y aproximación a los mitos y realidades del cine norteamericano destaca la pregunta que el científico Michio Kaku formula a sus lectores: “¿qué haríamos si internet un día 'despierta' y tienes planes diferentes a los del hombre?” (p. 108).

2 Resulta oportuno invocar la cuarta acepción del Diccionario de la Real Academia Española: "4. f. Psicol. Acto psíquico por el que un sujeto se percibe a sí mismo en el mundo”. Consultado el 3 de octubre de 2010 en: http://dle.rae.es/?id=ANy3DCx

3 European Research Council. "AI Makes Pop music in the style of any composer (The Beatles too!)", Flow Machines, consultado el 6 de octubre de 2016, disponible en: http://www.flow-machines.com/ai-makes-pop-music/

$4 \mathrm{Big}$ data es un término utilizado para referirse a un gran volumen de datos, generados y almacenados bajo las características de velocidad, variedad, complejidad y relevancia para un negocio en su operación del día a día. Ver, sas Institute Inc., "Big Data. What it is and why it matter", disponible en: http://www.sas.com/en_us/insights/big-data/ what-is-big-data.html. The Legal Black's Dictionary únicamente trae el término "Data", entendido como "el hecho que permite advertir una conclusión”. Consultado en línea el 6 de octubre de 2016 a través del vínculo http://thelawdictionary.org/data/ 
originales combinaciones de estilo, optimización e interacción de técnicas, así como componer en cualquier estilo" 5 . Tal progreso tecnológico permitió que el compositor, Benoit Carré (en adelante también denominado "el autor"), arreglara, produjera y escribiera las letras de la canción intitulada Daddy's Car, la cual cuenta con la particularidad de que fue "creada/fijada" a través de la plataforma Flow Machines y cuyo estilo pop tuvo inspiración en las características del cuarteto de Liverpool, The Beatles. Esta obra musical, entre otras, será publicada en un álbum cuyo lanzamiento se programó para inicios del año 2017.

La página web en comento planteó dos grandes preguntas: ¡cómo se hizo?, y ¿qué hizo la máquina y qué hizo el artista? Las respuestas a dichos interrogantes constituyen los pilares sobre los que se elaborará el presente ensayo. La productora señaló que:

1. Los desarrolladores crearon una base de datos denominada LSBD.

2. El humano compositor seleccionó un estilo y generó una partitura (melodía y armonía), a través de un sistema denominado Flow Composer. En particular, para la obra musical denominada Daddy's Car, el autor seleccionó el estilo de The Beatles.

3. A través del sistema llamado Rechord el músico humano compaginó algunos sonidos aislados de otras grabaciones para generar la partitura.

4. El músico humano finalizó la producción y la mezcla ${ }^{6}$.

Era un resultado programado que los diarios alrededor del globo y la cibersociedad especularían acerca de la protección de derechos de autor generados sobre la canción y acerca de la afectación en la esfera moral y patrimonial de los herederos y titulares, respectivamente. En particular, publicaciones periódicas en línea como $E l$ Mundo $^{7}$, La Tercera ${ }^{8}$ y $A B C^{9}$ han generado inquietantes reflexiones en torno al futuro de la industria musical, y en particular sobre la protección jurídico-autoral que recibirá la obra musical que evoca a The Beatles. El objetivo del presente ensayo es analizar las hipótesis legales que dentro del universo del derecho de autor efectivamente se pueden sostener, y descartar aquellas hipótesis que únicamente entorpecen el diálogo jurisprudencial.

5 Ibíd., 2.

6 Flow Machines. Artificial Intelligence for the future of music. SONY CSL Music, septiembre de 2016, versión completa en inglés disponible en: http://www.flow-machines. com/ai-makes-pop-music/ revisado el 01 de octubre de 2016.

7 Toledano, Bruno. "Una inteligencia artificial escribe una canción inspirada en The Beatles", El Mundo, España, 26 de septiembre de 2016, disponible en: http://www. elmundo.es/tecnologia/2016/09/26/57e93953e5fdea42278b4658.html

8 F.A.A. "The Beatles vuelve a la vida gracias a la Inteligencia Artificial", La Tercera, Madrid, septiembre 2016, disponible en: http://www.latercera.com/noticia/the-beatlesvuelve-a-la-vida-gracias-a-la-inteligencia-artificial/

9 Nieves, José Manuel. "Escuche la nueva canción de los Beatles creada por una Inteligencia Artificial", $A B C$, "Ciencia", Madrid, 27 de septiembre de 2016, disponible en: http://www.abc.es/ciencia/abci-escuche-nueva-cancion-beatles-creada-inteligencia-artificial-201609260853_noticia.html 


\section{OBJETIVOS A DESARROLLAR}

El presente trabajo de investigación está centrado en dos puntos fundamentales que nos permiten acompañar nuestras conclusiones y letras, a saber: 1) Se invocan antecedentes históricos sobre la interacción del hombre y la cibernética, en particular, sobre aquellas máquinas que permiten automatizar la información a partir de la introducción comercial de la red de redes. Se cierra dicha premisa con un breve análisis sobre el origen de la IA y cómo es que esta se ha concebido en su relación con el humano; y 2) Con fundamento en tratados internacionales administrados por la Organización Mundial de la Propiedad Intelectual y breves citas a la Ley Federal del Derecho de Autor de México, se precisan las figuras jurídicas, así como los límites que se encuentran en la legislación aplicable y que imposibilitan considerar a la iA como sujeto de protección autoral.

\section{EL JUEGO DE LA IMITACiÓN Y LA SEMÁNTICA JURÍDICA}

Detrás del gran avance en la automatización de la información y mecanización de procesos que ha llevado al hombre al progreso y evolución social, existen herramientas tan simples como el ábaco, las tablas de logaritmos (1614), la tarjeta perforada (1804), la máquina de Babbage (1834), hasta llegar a la MARK I o ASCC (siglas en inglés de Automatic Sequence Controlled Calculator), creada por la Universidad de Harvard con el apoyo de Iвм en 1937, la cual brindaba al usuario de la computadora electromecánica automática la posibilidad de obtener resultados de largas secuencias de operaciones codificadas, registrándolas en una cinta de papel perforada. Fue en 1963 cuando se introdujo por primera vez, al mercado de consumo popular, una computadora de tercera generación (predecesora de la ENIAC, la EDVAC y la UNIVAC, de 1943, 1945 y 1951, respectivamente) ${ }^{10}$ que permitió aumentar la velocidad y reducir los costos de producción, lo cual bien puede considerarse la revolución tecnológica que abrió paso a la era de la información, cuando en el año de 1969 se creó la primera conexión de interfaces, dando espacio a uno de los "servicios" de mayor consumo hoy en día, conocido como World Wide Web (www / Red de redes).

Más allá de los mecanismos computacionales, en el proceso de evolución tecnológica se desprende una rama que pretende brindar máquinas que eviten al hombre la operación de pensar, para generar un pensamiento más rápido y más exacto que el que cualquier hombre podría lograr ${ }^{11}$; fue en 1950 cuando el científico inglés

10 Téllez Valdés, Julio Alejandro. Derecho informático, México, Unam, 1987, consultado en línea el 26 de septiembre de 2016, disponible en: http://biblioteca.juridicas. unam.mx:8991/F/9L15JRAE4413579R4NR2F3CCEKGCRJ5YYMRJQH4H9X1QID7G 9D-28774?func=full-set-set \&set_number $=005726 \&$ set_entry=000001\&format $=999$

11 A mediados del siglo xx comenzó una guerra mediática y tecnológica entre la IA y la inteligencia mecánica/humana, en la cual se han obtenido resultados nada favorables para nuestra especie. Verbigracia, la computadora Deep Blue derrotó en el año de 1997 
Alan Turing hizo su ingreso al debate tecnológico con su artículo "Maquinaria computacional e inteligencia" ${ }^{12}$ y se tocó por primera vez el concepto de IA. Von Neumman continuó el trabajo de Turing, quien afirmó que las computadoras debían seguir un desarrollo de modelo similar al cerebro humano, ocupando conceptos de biología y anatomía humana, para referirse a las máquinas en aspectos relacionados con la "memoria" y los "sensores".

Empero, a finales de los años 50, los científicos McCulloch y Minsky construyeron un concepto opuesto, al afirmar que el proceso de gobierno de la información y las reglas que lo rigen deben ser distintas a las que se aplican como forma de gobierno del pensamiento y la materia; así las cosas, se abandonó la idea de que el cerebro de una máquina debía ser una réplica celular de lo que ocurre en la sinapsis humana ${ }^{13}$. A partir de esos postulados se celebró el primer congreso de la Universidad de Dartmouth relacionado con IA, del cual se extrajeron tres grandes principios nucleares de su método de estudio, a saber: 1) El pensamiento puede ocurrir fuera del cerebro humano, es decir, en máquinas; 2) El pensamiento puede ser comprendido de manera formal y científica; y, 3) La mejor forma de entender la IA es a través de las computadoras digitales ${ }^{14}$.

Los anteriores parámetros parecerían resolver la incógnita que años atrás planteara el científico inglés A. M. Touring, ya que de forma puntual desmitifican -de manera estandarizada para la comunidad científica- la exclusividad del pensamiento como característica particular de la especie humana, para compartirlo desde entonces con las máquinas. Así las cosas, aquello que comenzó como un

al maestro ajedrecista Garry Kasparov; Big Blue derrotó a un campeón de Jeopardy en televisión americana abierta, y en 2016 Alpha Go (de Google) venció de manera indiscutible al campeón de Go Lee Sedol. Hechos como los anteriores dejan serias dudas sobre la superioridad de los humanos sobre las máquinas y plantean el interogante de si estas ya han logrado una conquista sobre nuestra psique y sobre áreas en las cuales las capacidades cognitivas humanas parecerían insuficientes. Ver Lavenda, David, "The Battle of intelligence", Computer News Middle East (CNME), 22 de septiembre de 2016, consultado el 1 de octubre de 2016, disponible en: http://www.cnmeonline.com/insight/ the-battle-of-intelligence/

12 Turing, Alan, "Computing Machinery and Intelligence", Mind 49, 1950, disponible en: http://www.csee.umbc.edu/courses/471/papers/turing.pdf En el primer apartado de su texto, específicamente el parágrafo 1, “The Imitation Game”, Turing señala: "Propongo considerar la pregunta ¿pueden pensar las máquinas? Este sería el comienzo para definir el siginificado de términos como 'máquina' y 'pensar'. Las definiciones podrían ser calificadas como el reflejo de algunos usos consuetudinarios y normales, pero esta posición es peligrosa, pues si el significado de las palabras 'máquina' y 'pensar' puede ser examinado desde el punto de vista del uso común, entonces es difícil escapar a la conclusión de que el significado y la respuesta a la pregunta ‘¿pueden pensar las máquinas?' podría encontrarse en encuestas como las de la compañía Gallup Poll. Esto sería absurdo... ¿qué pasará cuando las máquinas tomen el rol del hombre en este juego?” (trad. libre).

13 ElgueA, Javier. Inteligencia artificial y psicología: la concepción contemporánea de la mente humana, Breve historia de la inteligencia artificial, México, Instituto Tecnológico Autónomo de México, 1987, disponible en: http://biblioteca.itam.mx/estudios/estudio/ estudio10/sec_16.html

14 Para mejores referencias, consultar la página conmemorativa Commemorating the 1956 founding ad Dartmouht College of AI as research discipline: http://www.dartmouth. edu/ -ai50/homepage.html 
juego de imitación en que el hombre pretendió crear máquinas con iguales y mejores características que las suyas, terminó siendo una realidad productiva, comercial e, inclusive, con trascendencia jurídica.

Más allá de los antecedentes tecnológicos e históricos de la IA, debemos buscar respuestas teleológicas y semánticas a las incógnitas formuladas en el presente trabajo de investigación. The Law Dictionary of Black define a la IA como "el software que se usa para permitir que computadoras y robots realicen trabajos mejor que el humano. El sistema se rige por conexiones neutrales. Es usada para ayudar a crear nuevos productos, robótica, entendimiento del lenguaje humano y visión de las computadoras"15.

\subsection{Implicaciones jurídicas en torno a la IA}

A la fecha de redacción del presente ensayo no se cuenta con un concepto que axiológicamente permita definir la IA, al existir tan diversas acepciones como ramas de estudio que se enfoquen a su definición. Dentro de aquellas que han pretendido aproximarse a la construcción de un concepto único, y con la humildad suficiente para comprender la volatilidad del término, cabe referirse al artículo "¿Qué es la inteligencia artificial?”, publicado por el Instituto Tecnológico Autónomo de México (IтAM) en 1987, el cual define a la IA como "cualquier máquina que desempeñe una función mental que tendría que ser realizada por una inteligencia humana"; asimismo, determina que la IA es un intento de reproducción de algunas características humanas; sin embargo, dentro de las conclusiones que se desprenden de tan digno trabajo de investigación, se abre la puerta para formular la teoría de existencia de inteligencia pura, es decir, independiente de cualquier voluntad o ejercicio racional de su creador.

Estos esfuerzos de construcción académica han demostrado que existen aceptaciones universales respecto del panorama de la IA en la jurisprudencia (léase ciencia jurídica), tanto en el modelo de superación de características humanas como en el reconocimiento doctrinal del pensamiento digital que les permitiría a las máquinas inteligentes ejecutar la tarea específica para la cual fueron concebidas; lo que, a su vez, permite limitar cualquier interpretación paranoica respecto del alcance de la conciencia artificial. En efecto, a diferencia de la libre y natural conciencia humana, la interpretación y racionalización de la existencia misma tiene tan diversas variaciones como personalidades y humanos vivan, en tanto que la conciencia artificial tendría su limitante, su ragion d'essere, en las instrucciones, modelos, algoritmos y lenguaje bajo el cual fue programado desde su nacimiento en el laboratorio de desarrollo.

Hasta este punto, la semántica jurídica e histórica-tecnológica nos permite afirmar que algunas máquinas tienen la facultad de pensar desde un punto de vista

15 "Artificial Intelligence", en The Law Dictionary of Black, consultado en línea el 29 de septiembre de 2016, disponible en: http://thelawdictionary.org/artificial-intelligence/ 
formal, en tanto que poseen la facultad pura y autónoma del hombre que las creó, a quien jurídicamente podemos identificar como productor, inventor, fabricante o desarrollador; sin embargo, dicho pensamiento y conciencia artificial encuentran las barreras tecnológicas en los alcances bajo los cuales fueron programados. Así es como se comprende la naturaleza y funciones de la plataforma Flow Machines, ya que su base datos y configuración únicamente genera obras musicales en términos de su código fuente, en atención a la programación detrás del software, lo que implica que la intervención de los programadores -en primera instancia- y del compositor que instruye a la plataforma -en segunda instancia- aún son de relevancia primaria y absoluta, sobre el mérito que constituye el medio informático en el que se plasma la obra musical. Hipótesis que podría verse destruida en un par de lustros, cuando las máquinas, efectivamente, logren obtener el libre albedrío que las detiene de la réplica humana exacta, con independencia de la superioridad mecánica que se les ha otorgado frente a la especie humana.

Los párrafos anteriores parecerían congruentes con el momento histórico y tecnológico que enfrentamos, sin embargo, el paradigma de que el "pensamiento" es exclusivo de la especie humana parece desaparecer cuando se estudia la naturaleza de la ia y las creaciones que pueden surgir de esta; en el entendido de que no solamente han logrado substituir al ser humano en las funciones que se les encomiendan, sino que han resultado superiores en diversos rubros productivos. A la vez, resulta justo afirmar que la construcción teórica y legal del derecho de autor tiene su base en un momento histórico que percibió remota la idea de creación fuera del razonamiento humano; a pesar de ello, a más de 300 años de distancia de la primera ley autoral ${ }^{16}$, sería prudente estudiar la posibilidad de adecuar conceptos tradicionalmente aceptados, que permitieran someter a debate público la presencia de conciencia artificial y pensamiento en máquinas de producción humana, cuya autonomía podría alcanzar el sello distintivo que hace tres siglos se creía exclusivo del hombre.

Conforme con los antecedentes descritos, así como con la construcción de los conceptos que se sujetan a consideración del lector y de las futuras generaciones de estudiosos de la IA, hemos delimitado lo que podría considerarse "sujeto de protección" para efectos de la ley; lo cual, sin duda, nos permitirá advertir si la IA debe contar con protección en materia de derechos de autor o bien considerarse "objeto de protección” y una herramienta mediante la cual los autores, artistas, intérpretes y ejecutantes, así como los productores, fijan sus ideas originales susceptibles de protección. Esto es, como una herramienta facilitadora de los derechos de autor. Bajo tal dicotomía, resulta prudente invocar los tratados internacionales que, administrados por la Organización Mundial de la Propiedad Intelectual

16 Departamento de Consultas, Dirección Jurídica, Instituto Nacional del Derecho de Autor. Estatuto de la Reina Anna 1710. 300 años de la promulgación de la primera Ley Autoral, Secretaría de Educación Pública, consultado en línea el 12 de noviembre de 2016, disponible en: http://www.indautor.gob.mx/documentos_principal/estatutoreina.pdf 
(OMPI), brindarán el panorama legislativo que hoy en día rige a las obras originales creadas con IA.

\title{
2. Consideraciones teóricas y Legales EN MATERIA DE DERECHOS DE AUTOR
}

La concepción subjetiva de creación y protección de derechos de autor ha determinado como pilar medular que únicamente las personas físicas son generadores de obras y sujetos de protección del sistema jurídico autoral que corresponda; así lo han confirmado la teoría, la jurisprudencia internacional ${ }^{17}$ y criterios de la OMPI; y de igual modo lo defienden expertos en la materia como Parets Gómez, quien en su Teoría y práctica del derecho de autor señala:

\begin{abstract}
Sólo el hombre es capaz de crear obras protegidas por el derecho de autor, en virtud de que goza de la capacidad humana para llevar a cabo creaciones de diversa naturaleza que de forma original se hacen susceptibles de la tutela jurídica y que es parte del proceso de creación intelectual... El sujeto principal del derecho de autor es el creador en su amplio sentido, toda vez que tal calidad jurídica no se adquiere en virtud de una obra determinada, sino como producto de la actividad humana originaria que fija ideas que adquieren una traducción material a través de una soporte básico sea cual sea su naturaleza ${ }^{18}$ (resaltado fuera de texto).
\end{abstract}

Concepción que también ha desestimado la posibilidad de que otro tipo de seres vivos pudieren llegar a generar derechos susceptibles de protección en el mundo autoral, tal como ocurrió en el juicio ventilado ante el Tribunal Federal de San Francisco y resuelto por el juez William Orrick, cuya sentencia expresa no conceder derechos de autor al mono Naruto sobre una fotografía tomada en forma de selfie, a pesar de las grandes demandas sociales pro animal y al limbo jurídico en que el dictamen final había dejado a la obra fotográfica ${ }^{19}$. Tales consideraciones y criterios internacionales parecerían impedir que cualquier ser ajeno a la naturaleza humana

17 Ver la acción de inconstitucionalidad ejercida por la Asociación de Compositores y Autores Musicales de Costa Rica ante la Sala Constitucional de la Corte Suprema de Justicia, exp. 95-003560-007-CO-C, que define la propiedad intelectual como la protección "a los intereses de los creadores de la obra, acordándoles derechos de propiedad sobre sus creaciones. La mayoría de las naciones han reconocido, en sus legislaciones internas, estos derechos de propiedad, con el objetivo de estimular la creatividad del intelecto humano". El extracto de la sentencia 0364-98 se toma de JimÉNez, Nidia Durán (Jueza Civil del Poder Judicial de Costa Rica, San José), Tercer Seminario Regional sobre Propiedad Intelectual para Jueces y Fiscales de América Latina. Organizado por la ompi, la Oficina Europea de Patentes, la Oficina Española de Patentes y Marcas, con la colaboración del Ministerio de Cultura de España, el Consejo General del Poder Judicial de España, la Agencia Española de Cooperación Internacional y la Secretaría de Integración Económica Centroamericana, OMPI/PI/JU/LAC/04/31, octubre de 2004, consultado en línea el 9 de octubre, disponible en: http://www.wipo.int/edocs/mdocs/mdocs/es/ompi_pi_ju_lac_04/ompi_pi_ju_lac_04_31.doc

18 Parets Gómez, Jesús. Teoria y práctica del derecho de autor, México, sista, 2012.

19 Allen Green, David. "Derecho de autor: no es momento de hacer el mono", Revista de la Organización Mundial de la Propiedad Intelectual, septiembre de 2014, disponible en: http://www.wipo.int/wipo_magazine/es/2014/05/article_0004.html 
sea originador de ideas susceptibles de alcanzar la protección del sistema autoral relativo, lo anterior bajo el argumento medular de que únicamente el ser humano es capaz de realizar un proceso intelectual que le permita plasmar una idea mediante un sello personalísimo e inimitable de su personalidad; sin que resulte óbice a lo anterior el referir que la IA cuenta con capacidades de "pensamiento" y conciencia artificial, ya que dichas características se encuentran limitadas a las instrucciones que de origen se han programado en su código fuente o formal; y asimismo, a la característica esencial de protección de las ideas que ocupan el mundo autoral, las cuales únicamente pueden ser producidas con las condiciones humanas antes referidas. De allí la imposibilidad metodológica y jurídica para calificar a la IA como sujeto de protección autoral -de origen-. En consecuencia sería prudente comenzar a estudiarla como el centro del objeto de la protección, esto es, la obra per se, y no como creador o titular de derechos de autor.

\subsection{Marco legislativo aplicable}

Sería inadecuado limitar los fines del presente trabajo de investigación a destruir las tendencias de las redes sociales y sus preocupaciones, sin brindar una real y aproximada respuesta jurídica a la interrogante: ¿qué derechos de autor se han originado con "Daddy's Car"? La cual podría encontrar luz en los ordenamientos internacionales siguientes: 1) el Convenio de Berna para la Protección de las Obras Literarias y Artísticas (en adelante "Convenio de Berna") ${ }^{20}$; 2) el Tratado de la OMPI sobre Derecho de Autor ${ }^{21}$, y 3) el Convenio de Roma sobre la protección de los artistas intérpretes o ejecutantes, los productores de fonogramas y los organismos de radiodifusión. Invocación que no atiende a un caprichoso silogismo que dependa de tales ordenamientos internacionales, sino al beneficio que pudiera generar en la interpretación de cualquier lector del presente ensayo; en el entendido de que las legislaciones locales podrían regular las figuras jurídicas que enseguida se estudiarán, siempre que cumplan con las características obligadas mínimas, establecidas por los mencionados tratados.

\subsection{Derechos de autor susceptibles de protección}

En primer lugar es pertinente recordar el concepto de "obra”, para después acotarlo a la hipótesis digital que hoy nos ocupa. Según la OMPI, se puede considerar obra a

20 Convenio de Berna para la Protección de las Obras Literarias y Artísticas, disponible en: http://www.wipo.int/wipolex/es/treaties/text.jsp?file_id=283694

21 El 20 de diciembre de 1996, la OMPI asumió la administración del Tratado de la OMpI sobre Derecho de Autor, que entró en vigor en marzo de 2002 y subsume la soberanía de 94 países contratantes (entre los cuales se encuentran grandes potencias creadoras de propiedad intelectual como Alemania, Estados Unidos, China y Japón, así como países en vías de desarrollo y que también han suscrito el Acuerdo de Asociación Transpacífico, como Chile, Colombia y México). Este se encuentra disponible en: http:// www.wipo.int/wipolex/es/treaties/text.jsp?file_id=295158 
toda creación original que se desarrolle en el ámbito del intelecto humano artístico y literario, tal como los libros, la música y arreglos musicales, la pintura, la escultura y las películas, los cuales se encuentran debidamente regulados en el artículo $2 .^{\circ}$ del Convenio de Berna ${ }^{22}$; asimismo, se considerará obra a los programas informáticos (también referidos como programas de computadora, programas de ordenador o software, con independencia de su modo de expresión), según lo dispone el artículo 4. ${ }^{\circ}$ del Tratado de la OMPI sobre Derecho de Autor; por último, las bases de datos, a pesar de no ser consideradas como una "obra" original strictu sensu, cuentan con la protección que brinda el artículo $50^{\circ}$ del mismo Tratado, suficiente (aunque no temporal como sí lo hace nuestra Ley Federal del Derecho de Autor) y generadora de propiedad -no intelectual- a favor del creador.

Una vez determinado el objeto de protección de las normas jurídicas, nos permitimos estudiar los conceptos jurídicos que brindan protección a los diversos autores dentro del caso de estudio:

- Derecho de autor sobre la obra musical. La obra cuyo estudio nos ocupa (Daddy's Car, "la obra") se considera una composición musical con letra en términos del artículo $2 .^{\circ}$ del Convenio de Berna. Lo anterior, en tanto que los incisos 1 y 3 protegen de forma puntual "las composiciones musicales con o sin letra" y "arreglos musicales", respectivamente. Lo anterior permite aseverar que los derechos de autor (morales y patrimoniales) pertenecen originariamente al autor. Sin que resulte óbice a lo anterior el medio a través del cual Benoit Carré hubiese generado los arreglos y la escritura de las partituras por medio de la plataforma Rechord, ya que el método de fijación no resulta un criterio de discriminación para conceder protección autoral al sujeto creador, en tanto que dicha acción ocurra sobre un soporte que permita la reproducción, percepción u otra forma de comunicación, tal como ocurre en nuestra hipótesis. Así lo indica la Ley Federal del Derecho de Autor, cuyo artículo sexto regula la fijación en los Estados Unidos Mexicanos como sigue: "Fijación es la incorporación de letras, números, signos, sonidos, imágenes y demás elementos en que se haya expresado la obra, incluyendo los electrónicos". Protección que se brinda a las obras materializadas siempre que cumplan con las características de percepción antes citadas. De tal suerte que es correcto afirmar la obra Daddy's Car se fijó sobre un soporte electrónico denominado Flow Machine.

-Derechos de autor sobre el programa de ordenador (software). Si bien el Tratado de la OMPI sobre Derechos de Autor no define el concepto de programas de ordenador, la Ley Federal del Derecho de Autor (México) se aproxima a una percepción que podríamos tomar como tradicionalmente aceptada, al definir a los programas de computación como "la expresión original en cualquier forma, lenguaje o código, de un conjunto de instrucciones que, con una secuencia, estructura y organización determinada, tiene como propósito que una computadora o dispositivo realice una 
tarea o función específica” ${ }^{23}$. Sin embargo, dicho Tratado, en su artículo $4 .^{\circ}$, dicta de forma puntual que los programas de ordenador alcanzarán la protección como obras literarias, cualquiera que sea su modo o forma de expresión, según lo dispone el artículo 2. ${ }^{\circ}$ del Convenio de Berna. Para acotar los alcances del presente estudio es prudente invocar el criterio del Doctor Pérez Miranda, quien invita a la prudente diferenciación entre un programa de cómputo y una obra literaria, no sólo sobre la máxima de que la ley lo distingue (a contrario sensu del principio Ubi lex non distinguit, nec nos distinguere debemus), sino sobre el elemento original, que invita a la prudente diferenciación entre un programa de cómputo y una obra literaria, toda vez que su alcance jurídico es diverso, en tanto que el elemento original de protección no recae sobre la programación per se, sino sobre el instrumento técnico y la forma en que este genera instrucciones ${ }^{24}$.

- Relación jurídica entre el autor y los desarrolladores. SONY CSL y la Universidad Pierre and Marie Curie de París, como comitentes de la obra, son los titulares de los derechos de autor (patrimoniales, con el debido reconocimiento de los derechos morales de los desarrolladores-personas físicas), en tanto que la persona física y autor, Benoit Carré, gozará de la protección de los derechos morales que las leyes le reconozcan. Así lo podemos afirmar, toda vez que la voluntad primigenia para la creación de la obra que hoy estudiamos surgió en la esfera de los comitentes, SONY CSL y la Universidad parisina, y Benoit Carré recibió la instrucción de generar la obra musical a través de la plataforma Flow Machine, por lo que se le denomina autor comisario, bajo la figura jurídico-autoral de obra por encargo, en estricta aplicación de los artículos 81, 83 y 83 bis de la Ley Federal del Derecho de Autor mexicana. Por lo anterior, se precisa que Daddy's Car es una obra por encargo, fijada en el soporte electrónico Flow Machine, cuya titularidad de derechos patrimoniales les pertenece a los desarrolladores. Hasta este punto, el software únicamente actúa como herramienta auxiliar de fijación de la obra Dadd'ys Car, y es por medio de ella que se obtiene la protección autoral.

- Base de datos. A pesar de que la Ley Federal del Derecho de Autor (art. 107), como el Tratado de la OMPI sobre Derechos de Autor (art. 5.o), realiza un ajuste legislativo, por analogía, de las bases de datos a las compilaciones (de datos), tratadistas del derecho de autor como los doctores Pérez Miranda y Parets Gómez han confirmado que resulta una calificación inadmisible una simple analogía con respecto a otras figuras de protección autoral, máxime siendo que las bases de

23 Art. 101 de la Ley Federal del Derecho de Autor (México), disponible en: http:// www.ordenamientojuridico.org. $\mathrm{mx}$

24 Tanto el artículo 102 de la Ley Federal del Derecho de Autor como el referido artículo $4 .^{\circ}$ del Convenio de Berna indican que los programas de cómputo deberán obtener la protección y el tratamiento de las obras literarias. PÉrez Miranda, Rafael Julio. Tratado de derecho de la propiedad industrial. Patentes, marcas, denominación de origen, obtentores de vegetales, informática. Un enfoque de derecho económico, 5. ${ }^{\mathrm{a}}$ ed., México, Porrúa, 2011, p. 391. 
datos comúnmente se encuentran ligadas a los programas de cómputo, para las cuales fueron creadas.

Con independencia de lo anterior, se advierte que el compilador -persona(s) física(s) o moral(es) - adquiere un derecho de naturaleza diferente a la de los derechos de autor, pero que sí vincula un derecho de propiedad sobre la compilación de la base de datos que nos ocupe. En el entendido de que únicamente se adquiere la protección respecto de los criterios y mecanismos de utilidad (respecto del software) de dicha base de datos, en tanto que los datos o materiales contenidos en la misma no se abrazan sobre la misma protección y protegen los derechos de autor que efectivamente pudieren surgir sobre cada uno de los elementos de la base datos. En concreto, se aduce que la $\angle S D B$ es producto de un trabajo de compilación digital para almacenar datos musicales en beneficio de la plataforma Flow Machine (software); estos derechos de propiedad, prima facie, pertenecen a la persona jurídica SONY CSL, sin que al respecto sea oportuno destacar de entrada la participación de la tecnología informática aplicada, si bien podría ser válido hacerlo en un secundo momento, ya que la herramienta sui generis bajo la cual se realiza el trabajo de compilación únicamente agrega un factor característico distintivo que vale la pena analizar de la forma en que se ha indicado.

Conforme lo anterior, sería inadecuado otorgar mayor mérito a la iA del que efectivamente merece, pues no se puede hablar de que el software "aprendió" dichas melodías, armonías y estilos, sino que resulta el trabajo de uno o varios programadores para almacenar obras jurídicamente protegidas. Esto implicaría que, de existir alguna reclamación autoral, podría proceder aquella relacionada con el derecho patrimonial (del que gozan todos los autores y/o titulares de tales facultades) de exigir una respuesta económica a la actividad de almacenamiento.

- Producción (arreglos). Parets Gómez define a los productores de fonogramas como "la persona física o moral que fija por primera vez los sonidos de una ejecución u otros sonidos o la representación digital de los mismos, siendo responsable de la edición, reproducción y publicación de fonogramas, de lo que se advierte que su labor esencial consiste en la actividad de fijación, pero no de cualquier creación artística, sino de sonidos de una ejecución u otros de carácter digital" ${ }^{25}$.

Por su lado, el Convenio de Roma define (art. 3. ${ }^{\circ}$ inc. c) que el productor de fonogramas es la persona natural o jurídica que fija por primera vez los sonidos de una ejecución u otros sonidos ${ }^{26}$.

Concepto teórico y concepto legislativo, respectivamente, que permiten comprender que la persona que efectúa una modificación a una obra original "para

25 Parets Gómez, Jesús. Teoría y práctica del derecho de autor. V. 2.2 Los productores de fonogramas, México, sista, 2012, p. 145.

26 Convenio de Roma, consultado en línea el 8 de octubre de 2016, disponible en: http://www.wipo.int/edocs/lexdocs/treaties/es/rome/trt_rome_001es.pdf 
embellecer la melodía"27 adquiere la titularidad de los derechos conexos (related rights), definiendo estos como las facultades con que cuentan los artistas, intérpretes, ejecutantes y productores de fonogramas sobre una obra musical protegida por el derecho de autor ${ }^{28}$. Bajo la metodología empleada para este trabajo de investigación, comenzaremos por definir los derechos conexos efectivamente protegidos en el caso de la obra, y posteriormente descartaremos las facultades incorrectamente atribuidas a la IA.

En un principio, no deseamos obviar que todo el proceso creativo autoral surgió bajo la figura de obra por encargo, a través de la cual la compañía sonY CSL y la Universidad parisina encomendaron al autor la creación de Daddy's Car. Conforme tal relación jurídica autoral, Benoit Carré escribió las letras y la música, definió la armonía y melodía a través de las partituras en Rechord, para finalmente fijar su obra, producirla y mezclarla a través del software. En principio, esto permitiría comprender que los derechos conexos de forma originaria podrían pertenecer a la compañía que comisionó la creación de la obra, salvo que existiere un pacto en contrario a favor del autor (persona física). El trabajo intelectual que ha realizado el compositor no sólo ha adquirido protección en la amplia esfera de los derechos de autor, sino de los derechos conexos, tal como se ha acreditado con anterioridad. En tales términos no resulta adecuado afirmar que la plataforma, Flow Machines, podría contar con derechos relativos a la producción del fonograma final, independientemente de las características bajo las cuales se ha concebido (programado), ya que esto implicaría subsumir cualquier proceso de "embellecimiento musical" en estas hipótesis tecnológicas, en el entendido de que los productores normalmente utilizan sofisticadas herramientas (tanto de hardware como de software) para brindar a la obra musical una nueva y final apariencia que beneficie su comercialización. Es decir, la IA no merece ser considerada titular de derechos conexos conforme tales consideraciones, sino como la herramienta/mecanismo que se utilizó para la fijación de la obra musical.

Los anteriores argumentos no pretenden afirmar de manera dogmática que la IA jamás estará en posibilidades de producir obras intelectuales, originales y cuyo mérito estético estñe dentro de la esfera de los derechos de autor; sin embargo, los silogismos antes expuestos permiten acreditar que la situación en contexto no merece un estudio adelantado de nuestra materia y que las figuras tradicionales, de derechos de autor, de derechos conexos y de derechos de propiedad sobre la base de datos, resultan aplicables a cada una de las situaciones de derecho que cabría encontrar dentro de las apresuradas y temerarias aseveraciones de los medios de comunicación.

27 “¿Qué es un arreglo musical?”, en Muy Interesante, consultado el 7 de octubre de 2016, disponible en: http://www.muyinteresante.es/cultura/arte-cultura/articulo/ique-esun-arreglo-musical

28 Instituto Ecuatoriano de la Propiedad Intelectual. ¿Qué es el Derecho de Autor y Derechos Conexos?, disponible en: http://www.propiedadintelectual.gob.ec/que-sonderechos-de-autor-y-derechos-conexos/ 
Objeto de un estudio diverso podría ser el de si "replicar" el estilo de un grupo musical sería causante de violaciones en materia de derechos de autor; sin embargo ello también implicaría descartar la posibilidad que podría existir de inspiración en los ídolos que impulsaron la carrera de tantos nuevos artistas, intérpretes y ejecutantes. Basta regresar un par de años en la historia musical para recordar que John Lennon ${ }^{29}$ y Paul McCartney confirmaron en diversas ocasiones su admiración e inspiración musical, en la época de de sus primeras obras, en el Rey del Rock, Elvis Presley ${ }^{30}$, o bien aquella influencia que marcaron las celosas enseñanzas de Mozart a favor del joven Beethoven; lo anterior, aunado a la imperante imposibilidad jurídica de proteger "estilos de composición y ejecución" como reserva de derechos en el marco de la ley federal autoral vigente ${ }^{31}$, y a que ninguna de esas conductas podría calificarse, en estricto sentido, como plagio. Destaca la sentencia 597/1997 del Tribunal Supremo de España que el plagio es:

... todo aquello que supone copiar obras ajenas en lo sustancial, por lo que se presenta como una actividad mecanizada, muy poco intelectual y creativa, carente de toda originalidad y concurrencia de genio o talento humanos, aunque manifieste cierto ingenio, dándose en la situaciones de identidad y en aquellas otras que, aunque encubiertas, descubren similitud con la creación original, una vez despojadas de ardides y disfraces, produciendo un estado de apropiación y aprovechamiento de la labor creativa y esfuerzo ideario o intelectivo ajeno.

No constituiría plagio, por el contrario, como recuerda la sTs de 20 de febrero de 1992,

... la mera confusión con todo aquello que es común e integra el acervo cultural generalizado o con los datos que las ciencias aportan para el acceso y conocimiento

29 Goldman, Albert. Las muchas vidas de John Lennon, Penguin Random House, 2010, p. 934. En esta obra biográfica se realiza un estudio sobre la influencia de Heartbreak Hotel (interpretada por Elvis Presley) sobre un solitario y desmotivado Lennon; en esta, como en otras biografías -incluidas las no autorizadas-, se recalca la función de catalizador mediático y musical que desempeñó el rey del rock para el líder de una de las bandas más populares de la historia. Disponible en: https://books.google.com.mx/ books?id=U3TrgG8FSnsC\&lpg=PT66\&ots=MBaKPdtlVm\&dq=entrevista\%20john\%20 lennon \%20 elvis\%20 presley $\& \mathrm{hl}=\mathrm{es} \& \mathrm{pg}=\mathrm{PT} 66 \# \mathrm{v}=$ onepage $\& \mathrm{q}=$ entrevista\%20john \%20 lennon\%20elvis\%20presley\&f=false Consultado el 7 de octubre de 2016.

30 No existen versiones oficiales sobre los encuentros entre The Beatles y Elvis Presley, sin embargo, en distintos diarios musicales se ha confirmado que los músicos mantenían comunicación por escrito.

31 La Ley Federal del Derecho de Autor, vigente en México, otorga la facultad de explotar y usar de forma exclusiva títulos, denominaciones, características físicas y psicológicas distintivas o características de operaciones originales aplicados a las publicaciones periódicas, difusiones periódicas, personajes humanos de caracterización, personas o grupos dedicados a actividades artísticas y promociones publicitarias; en el entendido de que dicha reserva no podrá ser un límite a la futura creación de obras originales ni un límite a la originalidad de los autores, en tanto que estos no violen las reservas de derechos ni derechos de autor previamente reconocidos. 
por todos. Por todo lo cual el concepto de plagio ha de referirse a las coincidencias estructurales básicas y fundamentales y no a las accesorias, añadidas, superpuestas o modificaciones no trascendentales ${ }^{32}$.

Argüir lo contrario podría conducir a un complejo examen subjetivo sobre cada una de las obras creadas y sobre la pureza plasmada en cada una de ellas; empero, siempre se debe observar el respeto a las obras ya concebidas y los derechos de autor que ello origine.

El progreso tecnológico y el paulatino avance legislativo en la regulación de la robótica y la IA (v.gr., Korean Law of Development and Distribution of Intelligent Robots y Legal Regulation of Autonomous Systems in South Korea) podrían ser hipótesis jurídicas que adquieran popularidad académica en un par de años; sin embargo, la IA ha acreditado que actualmente opera como una herramienta auxiliar en la prosecución de los diversos fines del ser humano y que, por ahora, opera bajo instrucciones programadas que no permiten atribuirle autonomía y conciencia, lo que nos llevaría a contemplarla como sujeto de derecho; por el contrario, su interoperabilidad con la sociedad únicamente le confiere el calificativo de objeto de derecho. Por ahora, el debate no permite analizar la personería de la IA y su equivalencia jurídica con la persona (física o jurídica), por lo que la conciencia artificial de Flow Machines podría "dormir tranquila" y saber que no se buscará reivindicar interés jurídico alguno por los herederos del cuarteto de Liverpool; por ahora, su contribución a la industria musical podría perderse como lágrimas en la lluvia.

\section{Conclusiones}

1. El progreso tecnológico ha permitido que el ser humano desarrolle y programe máquinas que pretenden substituir, al menos en igualdad de circunstancias, el desempeño humano.

2. La iA ha superado, en algunas ramas y sectores de la actividad mecánica humana, a sus creadores.

3. El concepto de pensamiento ha dejado de pertenecer de manera absoluta a la especie humana y es pertinente relacionar el mismo con la IA, para sintetizar el conjunto de actos que dichas máquinas son programadas para realizar.

32 El extracto citado corresponde a la sentencia 597/1997 del Tribunal Supremo español. El análisis de dicho criterio corresponde a Aragón, Emilia (Consejera Técnica del Ministerio de Cultura de Madrid), Tercer Seminario Regional sobre Propiedad Intelectual para Jueces y Fiscales de América Latina, organizado por la ompI, la Oficina Europea de Patentes, la Oficina Española de Patentes y Marcas, con la colaboración del Ministerio de Cultura de España, el Consejo General del Poder Judicial de España, la Agencia Española de Cooperación Internacional y la Secretaría de Integración Económica Centroamericana, OMPI/PI/Ju/LAC/04/18, octubre de 2004, consultado en línea el 9 de octubre, disponible en: http://www.wipo.int/edocs/mdocs/mdocs/es/ompi_pi_ju_lac_04/ ompi_pi_ju_lac_04_18.doc 
4. El conjunto de actos y resultados obtenidos por la debida operación de la IA puede ser considerado como conciencia artificial, aclarando que esta únicamente puede realizar lo que fue programado en sus circuitos y justo con el nivel de desempeño para el cual se le ha configurado. La conciencia artificial, a diferencia de la conciencia humana, carece de autonomía y libertad de pensamiento, reservadas para la especie humana.

5. En este momento y contexto jurídico-histórico no es posible afirmar que la IA no posee facultades para crear una obra original susceptible de protección autoral.

6. La obra musical Daddy's Car genera derechos de autor a favor de Benoit Carré, no sólo en la esfera de los derechos morales y patrimoniales, sino en la calidad de los derechos conexos. Asimismo, la compañía desarrolladora/productora sonY CSL y la Universidad Pierre et Marie Curie poseen la titularidad de los derechos conexos sobre los arreglos musicales de la obra.

7. A falta de disposición o manifestación expresa, se presume la comisión de "obra por encargo", tanto de la obra original denominada Daddy's Car como del trabajo de producción realizado con apoyo del software cuya titularidad le corresponde a SONY CSL.

8. La plataforma Flow Machine, que ha recibido el calificativo tecnológico de IA, constituye el objeto de protección autoral a favor de sus desarrolladores, en calidad de programa de cómputo.

9. La iA es la herramienta tecnológica que en el ámbito digital le permitió al autor crear, fijar y producir la obra musical de la forma en que se conoce.

10. La IA, a la cual indebidamente se pretende reconocer derechos de autor (sujeto de protección), es el objeto de protección de las leyes autorales y los tratados internacionales aplicables.

11. La base de datos (Big Data) LSBD es el almacenamiento digital que permitió al software realizar la mezcla y ejecutar la instrucción final del productor, cuyo resultado es la obra musical (con arreglos) Daddy's Care. Esta base de datos genera derechos de propiedad diferentes a los autorales, que merecen un estudio diverso a nuestra materia.

12. En la hipótesis que nos ocupa, resulta claro que la IA fue auxiliar en la persecución de los fines autorales, una plataforma con la cual se logró fijar la obra y su producción final; sin que sea prudente considerar que la IA forma parte del proceso intelectual que se analizó.

\section{Bibliografía}

“QQué es un arreglo musical?”, en Muy Interesante. Consultado en línea el 7 de octubre de 2016, disponible en: http://www.muyinteresante.es/cultura/artecultura/articulo/ique-es-un-arreglo-musical

"Big Data. What it is and why it matter", sAs Institute Inc., disponible en: http:// www.sas.com/en_us/insights/big-data/what-is-big-data.html 
Allen Green, David. "Derecho de autor: no es momento de hacer el mono", Revista de la Organización Mundial de la Propiedad Intelectual, septiembre de 2014, disponible en: http://www.wipo.int/wipo_magazine/es/2014/05/ article_0004.html

Aragón, Emilia (Consejera Técnica del Ministerio de Cultura de Madrid), Tercer Seminario Regional sobre Propiedad Intelectual para Jueces y Fiscales de América Latina, organizado por la OMPI, la Oficina Europea de Patentes, la Oficina Española de Patentes y Marcas, con la colaboración del Ministerio de Cultura de España, el Consejo General del Poder Judicial de España, la Agencia Española de Cooperación Internacional y la Secretaría de Integración Económica Centroamericana, OMPI/PI/Ju/LAC/04/18, octubre de 2004. Consultado en línea el 9 de octubre, disponible en: http://www.wipo.int/edocs/mdocs/mdocs/ es/ompi_pi_ju_lac_04/ompi_pi_ju_lac_04_18.doc

Commemorating the 1956 founding ad Dartmoubt College of AI as research discipline, disponible en: http://www.dartmouth.edu/ ai50/homepage.html

Convenio de Berna para la Protección de las Obras Literarias y Artísticas, disponible en: http://www.wipo.int/wipolex/es/treaties/text.jsp?file_id=283694

Convenio de Roma, consultado en línea el 8 de octubre de 2016, disponible en: http://www.wipo.int/edocs/lexdocs/treaties/es/rome/trt_rome_001es.pdf

Departamento de Consultas, Dirección Jurídica, Instituto Nacional del Derecho de Autor. Estatuto de la Reina Anna 1710. 300 años de la promulgación de la primera Ley Autoral, Secretaría de Educación Pública, consultado en línea el 12 de noviembre de 2016, disponible en: http://www.indautor.gob. $\mathrm{mx} /$ documentos_principal/estatutoreina.pdf

Diccionario de la Real Academia Española.

Diccionario Legal de Black.

Durán Jiménez, Nidia (Jueza Civil del Poder Judicial de Costa Rica, San José), Tercer Seminario Regional sobre Propiedad Intelectual para Jueces y Fiscales de América Latina, organizado por la OMPI, la Oficina Europea de Patentes, la Oficina Española de Patentes y Marcas, con la colaboración del Ministerio de Cultura de España, el Consejo General del Poder Judicial de España, la Agencia Española de Cooperación Internacional y la Secretaría de Integración Económica Centroamericana, OMPI/PI/Ju/LAC/04/31, octubre de 2004, consultado el 9 de octubre, disponible en: http://www.wipo.int/edocs/mdocs/mdocs/es/ ompi_pi_ju_lac_04/ompi_pi_ju_lac_04_31.doc

ELGUEA, JAVIER. Inteligencia artificial y psicología: la concepción contemporánea de la mente humana, Breve historia de la inteligencia artificial, Instituto Tecnológico Autónomo de México, 1987.

European Research Council. "AI makes pop music in the style of any composer (The Beatles too!). Flow Machines”, consultado el 6 de octubre de 2016, disponible en: http://www.flow-machines.com/ai-makes-pop-music/

F.A.A. "The Beatles vuelve a la vida gracias a la inteligencia artificial”, La Tercera, 
septiembre de 2016, disponible en: http://www.latercera.com/noticia/thebeatles-vuelve-a-la-vida-gracias-a-la-inteligencia-artificial/

Flow Machines. Artificial Intelligence for the future of music, sony CsL Music, septiembre de 2016, disponible en: http://www.flow-machines.com/ai-makespop-musicl

Goldman, Albert. Las muchas vidas de John Lennon, Penguin Random House, 2010, disponible en: https://books.google.com.mx/books?id=U3TrgG8FSns C\&lpg=PT66\&ots=MBaKPdtlVm\&dq=entrevista $\% 20$ john $\% 20$ lennon $\% 20$ elvis $\% 20$ presley $\&$ hl=es\&pg=PT66\#v=onepage\&q=entrevista $\% 20$ john $\% 20$ lennon $\% 20$ elvis $\% 20$ presley $\& f=$ false

Instituto Ecuatoriano de la Propiedad Intelectual. ¿Qué es el Derecho de Autor y Derechos Conexos?, disponible en: http://www.propiedadintelectual. gob.ec/que-son-derechos-de-autor-y-derechos-conexos/

KaKu, Michio. La física del futuro. Cómo la ciencia determina el destino de la humanidad y nuestra vida cotidiana en el siglo XXI, disponible en: https://issuu. com/mazzymazzy/docs/la_f_sica_del_futuro._michio_kaku.

Lavenda, David, "The Battle of intelligence", en Computer News Middle East (CNME), 22 de septiembre de 2016, consultado el 1 de octubre de 2016, disponible en: http://www.cnmeonline.com/insight/the-battle-of-intelligence/

Ley Federal del Derecho de Autor, disponible en: http://www.ordenamientojuridico.org.mx

Nieves, José Manuel. "Escuche la nueva canción de los Beatles creada por una inteligencia artificial", $A B C$, "Ciencia", Madrid, 27 de septiembre de 2016, disponible en: http://www.abc.es/ciencia/abci-escuche-nueva-cancion-beatlesCreada-Inteligencia-Artificial-201609260853_Noticia.Html

Parets Gómez, Jesús. Teoría y práctica del derecho de autor, México, sista, 2012.

Pérez Miranda, Rafael Julio. Tratado de derecho de la propiedad industrial. Patentes, marcas, denominación de origen, obtentores de vegetales, informática. Un enfoque de Derecho económico, 5. a ed., México, Porrúa, 2011.

Téllez Valdés, Julio Alejandro. Derecho informático, México, Unam, Instituto de Investigaciones Jurídicas, 1987.

Toledano, Bruno. "Una inteligencia artificial escribe una canción inspirada en The Beatles", El Mundo, 26 de septiembre de 2016, disponible en: http:// www.elmundo.es/tecnologia/2016/09/26/57e93953e5fdea42278b4658.html

TRATAdo de la OMPI SObre Derecho de Autor, disponible en: http://www.wipo. int/wipolex/es/treaties/text.jsp?file_id=295158

Turing, Alan. "Computing Machinery and Intelligence", Mind 49, 1950, disponible en: http://www.csee.umbc.edu/courses/471/papers/turing.pdf 